\title{
Les effets imprévus de la diplomatie américaine dans le cadre du congrès pour la liberté de la culture
}

\author{
Simon Perdrisat \\ perdrisat@gmail.com \\ https://orcid.org/0000-0002-2437-0386 \\ Université de Genève \\ 2016
}

La diplomatie culturelle peut avoir des conséquences inattendues. Nous allons nous intéresser ici aux circulations des idées et aux divergences entre l'Europe et les USA dans le cadre du Congrès pour la liberté de la culture. Ce congrès est considéré aujourd'hui par un historien de la CIA comme une des opérations de la guerre froide les plus audacieuses et efficace. ${ }^{1}$

Ce travail emprunte certains concepts de l'approche constructiviste des relations internationales en particulier la construction des identités et des intérêts étatiques. La théorie constructiviste en Relation Internationale, inspirée des théories interactionniste et constructiviste développées dans la deuxième partie du 20e siècle en science sociale, conçoit les relations internationales comme un projet en construction, un processus, plutôt que comme un état de fait. Les caractéristiques des relations internationales telles que nous les connaissons ne viendraient pas de "la nature humaine" ou de caractéristique intrinsèque à la politique internationale, mais seraient des constructions historiques et sociales. La théorie s'articule donc autour des affirmations suivantes: (1) les États sont les unités d'analyse principales pour la théorie politique internationale; (2) Les structures clés du système étatique sont intersubjectives plutôt que matériel; et (3) les identités et les intérêts étatiques sont en grande partie construits par des structures sociales, plutôt que par donner par des systèmes exogènes comme la nature humaine ou la politique interne. ${ }^{2}$

\footnotetext{
${ }^{1}$ Michael Warner, "Origins of the Congress for Cultural Freedom," Studies on Intelligence 38 (1995), https://www.cia.gov/library/center-for-the-study-of-intelligence/kentcsi/vol38no5/pdf/v38i5a10p.pdf.

2 Alexander Wendt, "Collective Identity Formation and the International State.," American
} 
Cependant, ce travail se limite à l'étude de cas de certains comportements des agents et ne cherche pas à prouver leur implication sur la scène internationale de manière systématique. J'aimerais avant tout mettre en avant la possibilité d'effets imprévus sans pour autant chercher à en apprécier leur fréquence et leurs implications concrètes et rendre sensible le lecteur à l'interdépendance des identités idéologiques et nationales et de certains effets de la diplomatie étrangère au travers de la diplomatie culturelle. Le terme « effet imprévu » est à comprendre en termes de développement d'idées qui sont ensuite à l'origine d'effets qui s'éloignent de l'objectif politique et stratégique initial. C'est donc une approche internationale voir transnational dans la construction d'identité.

Dans une première partie introductive, nous introduirons une définition de la diplomatie culturelle et la guerre froide comme guerre idéologique, suivie d'un bref passage sur le Congrès pour la liberté de la culture puis nous aborderons le cas spécifique du comité américain. Dans un deuxième parti, nous aborderons deux cas concrets. Premièrement, un échange de courriers entre François Bondy et Daniel Bell, puis la polémique qui suit la publication de l'article de Bertrand Russell sur Rosenberg dans le Guardian. Pour terminer, nous exposerons sans nous y attarder quelques autres actions du Congrès en rapport avec la politique américaine et nous terminerons par une conclusion autour de l'idée de "fausse réalité" et des dynamiques interne-externe en relation internationale.

\section{La culture comme l'ensemble des représentations mentales et des pratiques d'un groupe donné}

La diplomatie est l'art de parler même avec ces ennemis, mais aussi d'agir. La diplomatie culturelle est centrée autour de la culture. On peut relever deux grandes visions de ce qu'est la culture. Dans la première, la culture serait les productions symboliques «de l'esprit». Dans cette vision, la division entre « culture savante » et la « culture populaire» amène souvent une vision encore plus restreinte qui écarte la «culture populaire ». Pour la deuxième vision, la culture serait l'ensemble des représentations mentales et des pratiques propres à un groupe donné. On voit que cette dernière définition est beaucoup plus large. Il ne s'agit pas ici de choisir une «bonne » définition, la deuxième définition, qui englobe des phénomènes plus larges, permet d'aborder la réalité avec une théorie assez permissive pour pouvoir rendre compte de l'unicité des faits. Autrement dit,

Political Science Review 88, no. 02 (June 1994): 385, doi:10.2307/2944711. 
en abordant la question de la culture avec une vision qui englobe une grande variété de phénomènes, on limite le risque de passer à côté d'éléments que l'on aura filtrés a priori part une définition qui semble être plus précise, mais qui est aussi restrictive. Il me paraît également intéressant de garder à l'esprit la proposition faite par François Chaubet dans la conclusion de son livre:

“au-delà des deux définitions constamment opposées de la culture (définition humaniste contre définition anthropologique), ne faut-il pas [...], s'en remettre à une troisième approche concrète : la culture entendue comme processus d'interactions ?". 3

Nous pouvons donc dire que la diplomatie culturelle est une forme d'action volontariste mise en place par divers acteurs, qu'ils soient étatiques, non étatique, voire supranationale, qui cherche à diffuser des pratiques ou les productions des pratiques. La culture que l'on cherche à diffuser n'est pas nécessairement une forme de la culture nationale.

\section{Guerre froide, une guerre idéologique}

À la suite de la Deuxième Guerre mondiale, les États-Unis et l'URSS entrent dans un conflit qui durera près d'un demi-siècle: la Guerre froide. Plus largement, les régimes communistes et les démocraties occidentales s'opposent dans un monde devenu bipolaire. Pourtant ces deux blocs éviteront l'affrontement direct qui les aurait amenés à une destruction mutuelle.

Mais surtout, les États-Unis cherchent à endiguer le communisme. C'est une guerre idéologique plus que territoriale qui se joue sur plusieurs tableaux dans une course à la suprématie mondiale. Paradoxalement, on y voit naître la course aux armements nucléaires. La situation peut être résumée par l'expression de Raymond Aron devenue courante: "Paix impossible, guerre improbable".

L'une des formes d'affrontement se déroulera sur le terrain de la culture. Un important programme de diplomatie culturelle sera d'abord développé en URSS, puis au États-Unis lorsqu'ils chercheront à combler leur retard et à ne pas laisser un vide qui pourrait être exploitable par l'URSS.

\footnotetext{
${ }^{3}$ François Chaubet and Laurent martin, Histoire Des Relations Culturelles Dans Le Monde Contemporain, Armand Colin, 2011.
} 


\section{Le Congrès pour la liberté de la culture}

Le cas du Congrès pour la liberté de la culture est dans ce sens révélateur. L'on peut voir ce congrès comme une réponse directe au "congrès mondial des partisans de la paix" créé par l'URSS. Il apparaît clairement que les États-Unis veulent éviter de laisser un vide qui pourrait être alors exploité entièrement par l'URSS. De plus, de nombreux intellectuels européens et particulièrement en France sont alors sympathisants du communisme. Fonder le secrétariat du congrès à Paris a donc aussi comme objectif d'amarrer les intellectuels critiques.

Ce congrès a plus tard donné une image d'intellectuels manipulés, simples marionnettes aux mains de la CIA. En effet, il fut financé par l'agence de manière secrète jusqu'en 1966. Lors des révélations de ces financements, ce fut un choc pour nombre de ses propres membres tout comme pour l'opinion publique.

Pourtant, le congrès a entrepris plusieurs actions afin de dénoncer des politiques américaines jugées aller à l'encontre des valeurs du congrès. Certains événements vont mettre à jour une tension entre le Comité américain et les deux organes principaux du congrès, à savoir le secrétariat international et le comité exécutif basés à Paris. Cette divergence d'opinion sur certaines questions n'est pas propre au congrès pour la liberté pour la culture, mais est plutôt révélatrice d'une opinion publique européenne dont les sensibilités peuvent se révéler différentes de celle des États-Unis.

Cependant, en finançant le congrès pour la liberté de la culture, la CIA a donné des moyens à une organisation que l'agence ne peut pas contrôler totalement, et ceci pour plusieurs raisons. Premièrement, l'idéologie sur laquelle est basé le congrès pour la liberté de la culture est justement la liberté et particulièrement la liberté d'opinion. Mais surtout, la CIA agit dans l'ombre. Michael Josselson excepté, l'agent de la CIA qui créera et dirigera le congrès, les autres intellectuels y participant ne semblent pas être au courant du financement et, malgré certains bruits de couloir, pensent agir en toute autonomie dans le cadre du congrès. Même si Michael Josselson est connu pour avoir dirigé le congrès avec beaucoup d'énergie et une main de fer, il aurait été difficile, sur certains cas dérangeants, d'être contre la liberté d'opinion sans créer des conflits qui auraient été certainement contre-productifs en termes de diplomatie culturelle. Ce genre de 
dérapage aurait certainement été récupéré par les communistes afin de décrédibiliser les États-Unis.

\section{Le comité américain, un cas particulier}

Le Comité for Cultural Freedom (CFF) fut fondé en 1939, en 1951 Sidney Hook réorganise le CFF en l'Américain Comitte for Cultural Freedom en conséquence de l'intérêt porté à la contre-démonstration à la Freedom House to the Communist Waldorf Peace Conferenc. ${ }^{4}$

Le Comité Américain, est le seul comité qui précède la naissance du Congrès pour la liberté de la culture et qui était, strictement parlant, un membre indépendant. ${ }^{5}$ Cependant, le comité américain sera hiérarchiquement subordonné au Congrès international. Cette position semble avoir créé un ressentiment profond qui fut accentué par le fait que le comité américain, contrairement aux autres comités nationaux, n'était pas financé par le Comité international. ${ }^{6} \mathrm{La}$ raison avancée était que la liberté de la culture semblait moins en danger aux États-Unis que dans d'autres régions du monde. Aussi le comité américain avait un meilleur accès à des sources de financement privé que d'autres comités nationaux.

La plus grande difficulté qu'a eue le Comité Américain fut les intérêts politiques divergeant de ses membres. Sur des questions spécifiques, l'intérêt commun pour les valeurs démocratiques n'était pas toujours suffisant pour développer une politique commune. Le plus grand clivage se produit autour du Senator Jose McCarthy et de ses campagnes contre la subversion communiste, spécifiquement en ce qui concerne les procédures d'investigation de la commission qu'il dirigeait. ${ }^{7}$

Le Comité Américain cesse toute activité en 1957 suite à des divergences internes et avec le Congrès et à ces problèmes financiers.

\footnotetext{
${ }^{4}$ Sidney Hook, Out of Step: An Unquiet Life in the 20th Century, 1987, 420.

${ }^{5}$ Ibid., 423.

${ }^{6}$ Hugh Wilford, The New York Intellectuals: From Vanguard to Institution (Manchester Univ Pr, 1995), 208.

${ }^{7}$ Sidney Hook, Out of Step: An Unquiet Life in the 20th Century, 422.
} 


\section{François Bondy en désaccord avec le comité américain}

François Bondy, journaliste suisse, est engagé par le congrès en 1950 afin de créer une revue francophone dont le but premier est avant tout de contrer Les Temps Modernes alors dirigés par Jean-Paul Sartre. Sous sa direction, le premier numéro de la revue Preuve est publié en octobre $1951 .^{8}$

Le 25 juin 1953, François Bondy écrit à Daniel Bell alors membre du comité américain un courrier dans lequel il exprime ses craintes et désaccords avec les actions du comité américain. Il précise qu'il parle en son nom, mais souligne que ses sentiments sont partagés par de nombreux Européens dont la plupart des contributeurs de Preuves. De manière générale, il critique le comité américain pour n'être pas assez actif dans les problèmes liés à la justice, à l'humanité et à la liberté qui ne sont pas directement liés à la lutte anticommuniste.

Dans ce courrier, Bondy critique notamment l'absence de déclaration du comité contre l'élimination des livres "d'auteurs controversés" des bibliothèques américaines. Surtout que le général Eisenhower avait donné un discours défendant le libre accès au livre au Dartmouth College: « Don't join the book burners. Don't think you are going to conceal faults by concealing evidence that they ever existed. Don't be afraid to go in your library and read every book, as long as that document does not offend our own ideas of decency. That should be the only censorship. $»{ }^{9}$ L'élimination des livres s'inscrit dans la période maccartisme. La période dite maccarthyste commence quand le sénateur Joseph McCarthy se lance en 1950 dans une politique qui consistait à déloger des éléments subversifs au sein du gouvernement américain et se termine par un vote de censure à l'encontre du sénateur en 1954. McCarthy assura posséder une liste contenant le nom d'employé de l'État américain affilié aux partis communiste. Par la suite, la commission dirigée par McCarthy s'attaqua au Overseas Library Program, le commission identifia et interdit plus de 30’000 livres pensés être écrit

\footnotetext{
${ }^{8}$ Frances Stonor Saunders, The Cultural Cold War: The CIA and the World of Arts and Letters (New Press, The, 2013), 85.

9 "Dwight D. Eisenhower: 'Remarks at the Dartmouth College Commencement Exercises, Hanover, New Hampshire.,"” June 14, 1953, http://www.presidency.ucsb.edu/ws/?pid=9606.
} 
pas des sympathisants communistes. De nombreuses bibliothèques aux ÉtatsUnis supprimèrent également ces livres de leurs collections. ${ }^{10}$

Notons que le discours d'Eisenhover fut accueilli avec enthousiasme par l'American Library Association qui s'en inspira pour écrire le Freedom to Read Statement. Cette déclaration défend un accès sans compromis à l'ensemble des livres: "The freedom to read is essential to our democracy. [...] Most attempts at suppression rest on a denial of the fundamental premise of democracy: that the ordinary individual, by exercising critical judgment, will select the good and reject the bad. We trust Americans to recognize propaganda and misinformation, and to make their own decisions about what they read and believe.". ${ }^{11} \mathrm{Il}$ deviendra rapidement un des principes fondamentaux des services de libraire. ${ }^{12}$

Il s'attache ensuite à l'affaire Rosenberg que nous allons aborder en détail par dans le deuxième cas. En effet, le comité américain n'est pas intervenu alors qu'en Europe l'affaire était perçue comme une problématique morale extrêmement importante. Il rajoute que l'empressement avec lequel la sentence de mise à mort a été exécutée a choqué l'opinion publique européenne.

Mais François Bondy n'est pas seulement en désaccord sur l'absence de déclaration du comité américain. Dans certains cas où le comité s'est exprimé, il en critique la forme. Il prend par exemple le cas de l'Afro-Américain Willie MacGhee jugé pour viol et condamné à la peine capitale. En effet, la culpabilité de MacGhee a fait débat aux États-Unis et en Europe deuxièmement aucun blanc n'avait jamais été exécuté pour viol au Mississippi. ${ }^{13}$ En France, l'exécution fut vivement critiquée par le public et le gouvernement. ${ }^{14}$ Le comité exécutif avait demandé un article au comité américain pendant plus de deux ans, mais l'article rendu s'attardait sur l'horrible aspect du crime de viol et avait intégré des aspects anticommunistes qui avaient déplu au comité central. Il en va de même pour la

${ }^{10} \mathrm{~K}$. Lee Lerner and Brenda Wilmoth Lerner, eds., Encyclopedia of Espionage, Intelligence, and Security - Volume 2, vol. 2 (Detroit: Thomson/Gale, 2004), 251.

11 American Library Association and American Book Publisher Council, "The Freedom to Read Statement," 1953.

${ }^{12}$ Wayne A. Wiegand, "The Freedom to Read - The History of ALA's Vital Statement on Intellectual Freedom,” American Libraries, March 15, 2016.

13 "Campaign in U.S. to Pardon Negro," The Manchester Guardian, May 5, 1951.

14 "Negro's Execution in Mississippi," The Manchester Guardian, May 10, 1951. 
résolution sur Chaplin, par laquelle Chaplin s'était vu privé du droit de revenir aux États-Unis.

Enfin, Bondy exprime son incompréhension quant au fait que le comité américain parle du devoir de dénoncer les communistes, dans le cas d'Einstein par exemple, sans parler des graves problèmes des procédures inquisitionelles et du manque de cadre légal. Il prend comme exemple le New York Times qui publia une demi-page de nom de personnes suspectées de communistes sur la base d'un témoin unique. Bondy, mets en avant les possibilités de chantage.

De manière générale Bondy critique le maccarthysme, pour Bondy, un tel climat ne peut pas être propice à la liberté de la culture.

Dans sa réponse datée du 29 juin 1953, Daniel Bell manifeste avant tout son accord avec la ligne politique exprimée par François Bondy. Il précise cependant que certaines actions du comité américain allant dans le sens réclamé par Bondy n'ont pas trouvé d'écho dans la presse, argumentant que les médias américains ne publient que les textes qui vont faire controverse. Il souligne que le comité américain ne dispose pas, contrairement à la France, de son propre organe de presse susceptible de mieux diffuser ses prises de position, ce qui est étrange, car le comité américain disposait de lien très étroit avec des publications comme le journal New Leader ou la revue Partisan Review. Mais Sidney Hook affirmera dans sa bibliographie que "Si techniquement le comité [américain] était l'éditeur de Partisain Review, sa ligne éditoriale en était complètement indépendante. La relation était plus celle d'un service de comptabilité permettant à Partisan Review de profiter du statut d'exonération de TAX du comité". ${ }^{15}$

Il admet cependant l'existence d'un problème politique au sein du comité américain, fractionné en deux groupes idéologiques: un groupe libéral qui ne souhaite pas être anticommuniste, mais antisoviétique, et un groupe aux vues anticommunistes plus radicales. La division entre les deux groupes est identifiée comme étant liée à McCarthy: personne ne lui étant favorable, le deuxième groupe s'inquiète de ce qu'une opposition directe à McCarthy ne soit utilisée par les communistes afin de nourrir la propagande communiste. La manière sont s'est

${ }^{15}$ Sidney Hook, Out of Step: An Unquiet Life in the 20th Century, 430. 
déroulé l'enquête du service Voice of America (VOA) a clairement démontré le danger représenté par McCarthy. Même si aucune charge n'est retenue contre le personnel de VOA, il est mis en accusation devant les caméras de télévision, les sous-entendus de McCarthy poussent tout de même un ingénieur au suicide. ${ }^{16}$

Bell propose d'envoyer à Paris toutes les copies des prises de position publiques du comité américain. Cette proposition peut être interprétée comme une tentative de délégation de la responsabilité du comité américain auprès du comité exécutif, Bell ne voulant pas risquer sa réputation. Alternativement, Bell étant plutôt sympathisant du groupe de gauche du comité américain et se sent donc proche des la position défendue par Bondy, proposer une relecture à Paris lui donne un avantage pour défendre ses positions dans le comité américain.

Sur de nombreux cas - Einstein, Rosenberg, MacGhee - Daniel Bell tente de nuancer les critiques de Bondy en argumentant d'une part de la complexité des cas, mais surtout, d'autre part, leur utilisation en tant que propagande communiste dans un but antiaméricain en servant d'étendard afin de lever des fonds conséquents pour les communistes. Enfin, il souligne le manque de moyen du comité américain pour justifier de ses limites et sollicite des ressources financières de Paris. François Bondy lui répondra que le problème lui paraît moral et intellectuel plutôt qu'organisationnel ou financier.

Dans ces échanges de courriers, de profondes divergences d'opinion entre Amérique et Europe et donc entre les deux comités sont manifestes. On retrouve dans la presse des deux continents ces divergences d'opinions, cependant il n'y a pas d'unanimité comme le confirment les lettres du lectorat qui témoigne de la réalité plus complexe avec des clivages moins nets. On peut citer par exemple l'organisation de campagne aux États-Unis appelant au pardon de MacGhee ${ }^{17}$ ou encore une lettre paru dans le Manchester ${ }^{18}$ critiquant fermement la position de Russell. Le comité américain en tant que groupe anticommuniste s'est laissé entraîner par ce qui prend de plus en plus la forme d'une psychose collective mccarthienne. Bien que Daniel Bell s'en défende, et justifie un certain nombre

${ }^{16}$ Christel Lamboley, Le Maccarthysme Ou La Peur Rouge: La Croisade Américaine Contre Le Communisme (50 Minutes, 2015), 25.

17 "Campaign in U.S. to Pardon Negro."

18 Wade . Mack., "Letters to the Editor - The Case Of Morton Sobell," The Manchester Guardian, April 3, 1956. 
des actions et prises de position du comité, il est probable qu'une critique émanant du comité central, bien que présenté à titre personnel, ait eu un impact et suscité une certaine remise en question.

En revanche, on peut noter une contradiction majeure entre une idéologie libérale exportée des États-Unis vers l'Europe, et ses limites lorsqu'il s'agit aux États-Unis d'éviter à tout prix que cette idéologie puisse être instrumentalisée par les mouvements communistes. Cette crainte va inhiber certaines prises de position et mener à une forme d'autocensure ${ }^{19}$ qui semble incompatible avec l'idéologie de la liberté de la culture.

\section{Bertrand Russell attaqué par le comité américain pour sa défense du cas Rosenberg}

Le deuxième cas auquel nous allons nous intéresser est celui de Bertrand Russell. Le mathématicien et philosophe était dans les années 1950, année où il reçut le prix Nobel, très célèbre. Il était l'un des membres honoraires du Congrès. Cependant, ses idées politiques pouvaient changer rapidement. Par exemple, alors qu'en 1948 il proposait de menacer les Soviétiques avec la bombe, au milieu des années 1950 il insistait sur l'importance d'un désarmement. Ces changements rapides créèrent de nombreux soucis au Congrès. ${ }^{20}$ Lors de plusieurs affaires, Russell menace de démission et en 1956, avec le cas de Morton Sobell, il passa finalement à l'acte. C'est ce dernier cas que nous allons étudier.

L'affaire Rosenberg est une accusation d'espionnage contre Julius et Ethel Rosenberg ainsi que leurs complices. Au moment où l'affaire éclate, de nombreuses personnes ont maintenu qu'ils étaient innocents et victimes de la paranoïa et de "chasse aux sorcières" liées à la guerre froide durant l'époque McCarthy. Cependant, la déclassification des archives de l'URSS désignera clairement Julius Rosenberg comme agent. Ethel Rosenberg semble en revanche

${ }^{19}$ Par exemple, le comité américain n'osant pas critiquer le maccartisme publiquement ou certaine bibliothèques se trouvant États-Unis qui supprime des livres de leur rayon à la suite de la liste étaient destinés aux bibliothèques de l' Overseas Library Program

${ }^{20}$ Saunders, The Cultural Cold War. 
avoir été condamnée sans réel motif, et ses descendants demandent aujourd'hui encore son pardon. ${ }^{21}$

L'opinion publique européenne fut en grande partie choquée par cette affaire, qu'elle suivit de près dans ses rebondissements. Le 13 mars 1956, Rose Sobell, mère de Morton Sobell, condamné comme complice dans l'affaire Rosenberg, s'était rendue à une réception à Manchester organisée pour elle par le "Manchester Roseberg-Sobell Comittee" où elle demanda aux Britanniques de faire pression sur le président américain Eisenhower afin de faire libérer son fil. ${ }^{22}$ Bertrand Russell fait paraître une lettre ouverte dans le Manchester Guardian le 26 mars 1956, dans laquelle il appelle l'opinion publique à soutenir une action en faveur de Morton Sobell. ${ }^{23}$ La lettre accuse le FBI d'avoir organisé l'enlèvement et la déportation de Sobell alors qu'il se trouvait au Mexique, et ce en toute illégalité. Elle dénonce les techniques d'enquête du FBI en les comparants à celles utilisées dans d'autres états totalitaires comme l'Allemagne Nazie et la Russie stalinienne:

"The technique is one with which we have been made familiar in other police States such as Nazi Germany and Stalin's Russia. The police find a man whom they can prove to be guilty of some offence and the promise him immunity if he will manufacture evidence against people who could not otherwise be indicted. Perjury is especially useful as a lever because many people who have been Communist in their student days rashly hope that this can be concealed and swear they were never Communist. After a sufficient number of secret interviews, the FBI descends upon innocent people with a posse of terrified perjurers and in the general hysteria every word uttered by the perjurers is accepted as gospel truth."

Le contenu de la lettre fut rapidement relayé dans la presse américaine et mondiale, notamment dans le New York Times. ${ }^{24}$ Rapidement, des critiques quant à son analyse furent publiées, par exemple dans le Washington Post, mais aussi dans le Manchester Guardian. ${ }^{25}$ Par la suite le sujet sera relancé à de multiples

${ }^{21}$ Michael Meeropol and Robert Meeropol, “The Meeropol Brothers Exonerate Our Mother, Ethel Rosenberg," The New York Times, August 15, 2015.

22 “Appeal For Morton Sobell," The Manchester Guardian, March 14, 1956.

${ }^{23}$ Bertrand Russell, "Letters to the Editor - The Sobell Case," The Manchester Guardian, March 26, 1956.

24 "Bertrand Russel Accuses the FBI Of Atrocities, Doubts Rosenbergs' Guilt," The New York Times, March 26, 1965.

25 "Bertrand Russell Criticised," The Manchester Guardian, April 3, 1956; Wade . Mack., "Letters to the Editor - The Case Of Morton Sobell"; "Russell Is Disputed," The New York 
reprises, notamment par Sartre le 15 juin dans le New York Times, faisant lui aussi mention des irrégularités de la procédure. ${ }^{26} \mathrm{Il}$ est particulièrement intéressant de relever la manière dont certaines figures intellectuelles alors en vue s'expriment largement sur le sujet, alors qu'elles n'apportent que rarement des éléments nouveaux. On a donc affaire à un sujet polémique, quand bien même un nouveau procès semblait fort improbable, car il aurait remis en question le système judiciaire américain et aurait alors touché à sa légitimité.

Dix jours après la publication de la lettre de Russell, le Comité américain publie un communiqué le blâmant fortement. Dans sa déclaration, le comité américain "ne dénie pas le droit d'exprimer une opinion, même si nous la considérons comme complètement erronée", mais il critique vivement "pour un ami de la liberté de la culture [..] d'émettre des déclarations fausses et non fondées sur les procédures judiciaires pratiquées aux États-Unis". De plus il souligne l'absence de preuve amenée par Russell pour justifier ses arguments. Mais surtout, il critique "les comparaisons que vous faites entre les procédés employés par le FBI et les méthodes policières de l'Allemagne nazie ou de la Russie stalinienne". Pour le comité américain, la lettre de Russell est un service rendu à l'ennemi. En réaction, Russell écrit à Stephen Spender, membre du Comité exécutif international pour lui indiquer son intention de démissionner.

Cette action du Comité américain va pour le moins irriter le Comité international. Premièrement, le communiqué fut publié sans s'en référer au Comité international. De plus, Michael Josselson avait demandé à Irving Kristo de trouver un correspondent Américain à Londres pour interviewer Russell dans le but de "montrer que Russell n'a aucune preuve et que sa déclaration était basée sur de la propagande communiste et que, au vu de sa sénilité, il ne lui était plus possible de distinguer de la vérité". ${ }^{27}$ Le Comité exécutif se rassembla en réunion extraordinaire où il vote une lettre de blâme dans lequel fut exprimée leur incompréhension fasse à une attaque si directe et personnelle alors qu'ils auraient simplement pu se désolidariser publiquement du point de vue de Bertrand Russell. Pour finir, il ordonnait au comité américain de passer dès lors par une

Times, April 8, 1956.

${ }^{26}$ Max Freedman, “The 'innocense' of Sobell - M. Sartre Gives His Views," The Manchester Guardian, June 16, 1956.

${ }^{27}$ Saunders, The Cultural Cold War, 194. 
validation auprès du Comité international pour toute affaire pouvant avoir des répercussions internationales. En résumé, il censurait de fait le comité américain. ${ }^{28}$

Pour comprendre la rage de Josselson, il faut savoir qu'il avait en 1954 coupé les subventions du Comité américain afin qu'il soit dans l'incapacité de poursuivre ses activités "more convinced than ever that a natural death of the present American Comittee would be the best things to happen to everyone concerned". ${ }^{29}$ Cependant, le comité américain en s'adressant directement à Allen Dulles avait reçu des fonds de la CIA sans que Josselson n'ait son mot à dire.

Josselson voulait la mort du Comité américain, car il s'éloignait du but original du Congrès en tant que défenseur de la liberté de la culture, l'encouragement de la recherche intellectuel et la promotion de la créativité pour se tourner principalement vers une politique anticommuniste et la défense des intérêts américains. Il faut dire que le climat du milieu des années 1950 était particulièrement peu propice à la tolérance et à l'ouverture d'esprit. On voit apparaître aux États-Unis une sorte d'absolutisme politique, qu'il soit d'obédience McCarthyiste, libérale, anticommuniste ou anti-stalinisme. Cet absolutisme pousse à créer de fausses vérités, à ignorer l'histoire ou exposer les faits de manière à les rendre compatibles avec une certaine idéologie politique. ${ }^{30}$ De nombreux intellectuels "combattant de la guerre froide" avaient une pensée qui se construisait principalement en étant le porte-à-faux de la conception qu'ils se faisaient du communisme. George Urban, directeur de radio Free Europe interrogé sur "la relation de contrepoint avec le communisme" de ses intellectuels conclut dans sa réponse:

"compulsion to argue, fence, and fight, almost regardless of the objectives... Their protestations were too intense, their cynicism too stark, and their analyses too reflective of the world they thought they had left behind. They marched in negative step, but in step all the same."

La discorde entre Russell et le Comité américain se poursuivra sur plusieurs courriers. Russel exigera finalement que le Congrès se dissocie publiquement de

\footnotetext{
${ }^{28}$ Ibid.

${ }^{29}$ Ibid., 193.

${ }^{30}$ Ibid., 192.
} 
l'action du Comité américain dans le cas Sobell. "À mon avis", déclara-t-il, "une organisation qui soutient ouvertement l'action de la justice dans le cas RoserbergSobell n'a pas le droit de se prétendre partisan de la liberté de la culture." Le Congrès envoya un projet de lettre qui devait être publié dans le Manchester Guardian, mais Russel ne le trouva pas adéquat. En effet, le projet de lettre s'abstenait ouvertement de critiquer le fond du problème soulevé par le cas Rosenberg, mais désapprouvait "la forme polémique adoptée par le Comité américain et qu'il se dissocie par conséquent de son action.". Russel finalement démissionna. Début 1957, le Comité américain avait également cessé d'exister pour cause de problème de financement.

Il me semble important de relever la rapidité avec laquelle les informations s'échangeaient à cette période. Il ne faut moins de 24 heures pour qu'un événement en Europe soit connu du public aux États-Unis et vice-versa. Bien que les techniques contemporaines de transmissions de l'information soient plus rapides, la diffusion de l'information au sein de la population n'apparaît pas significativement plus rapide aujourd'hui. Le rôle des journaux était à l'époque très important. Mais cette marée d'informations contradictoires, de communiqué, de lettre ouverte, de réponse, de contre arguments cherchait certainement moins à convaincre leur destinataire ou adversaire qu'à influencer le public. En effet, à cette période, il devient clair que l'on choisit une politique et que l'on agit en conséquence plutôt que de chercher des politiques par apport à une expérience réelle du terrain. Ces différents systèmes, en réagissant constamment à l'autre idéologie, même s'il y a une absence de dialogue constructif, deviennent tributaires du discours et du cadrage de l'idéologie adverse. L'élément intersubjectif d'identités sociales peut être coopératif ou conflictuel, ce qui importe c'est de voir à quel point les structures sociales qu'elles instancient pénètrent le concept de soi, et non pas s'il y a une intégration normative entre soi et les autres. ${ }^{31}$ La psychose est parfois telle que les individus cherchent l'idéologie adverse à l'intérieur de ce que l'on a défini comme son propre camp! Le discours et ses effets deviennent dès lors très difficiles à juger, les acteurs de ces polémiques ne cherchant plus vraiment à argumenter (personne n'apporte jamais de preuve), mais plutôt à choisir une sélection de faits qui permettent de marquer

${ }^{31}$ Wendt, "Collective Identity Formation and the International State.," 386. 
un point ou de décrédibiliser l'adversaire. Le but n'est plus de connaître la vérité, mais de vaincre.

Les journaux, en donnant la parole à des intellectuels engagés, communiqua une vue très contrastée des idées ce qui amplifia certainement le phénomène. Par exemple, à en croire la presse de l'époque, pour certains les États-Unis seraient un état policier de non-droit alors que pour d'autres les États-Unis seraient envahis par des communistes qu'il faudrait découvrir par tous les moyens et mettre hors état de nuire.

Went, argumente que Gorbachev termina la guerre froide en repensant ce qu'était la relation entre US-Soviet:

"Gorbachev's New Thinking was a deep, conceptual reassessment of what the US-Soviet relationship "was." It was constitutive theorizing, at the lay level, and based on it the Soviets were able to end, unilaterally and almost overnight, a conflict that seemed like it had become set in stone. It may be that objective conditions were such that the Soviets "had" to change their ideas about the Cold War, but that does not change the fact that in an important sense those ideas were the Cold War, and as such changing them by definition changed the reality." 32

\section{Autres actions}

En plus des deux cas que nous venons de voir en détail, voici encore quelques exemples tirés d'action principalement entreprise par le Congrès Internaternatonial et liée à la politique américaine:

- Protestation auprès de la Cour Suprême des États-Unis contre la sentence de mort prononcée à l'encontre de Willie McGee.

- Protestation contre l'admission de l'Espagne à l'UNESCO.

32 Alexander Wendt, Social Theory of International Politics, Cambridge Studies in International Relations 67 (Cambridge, UK ; New York: Cambridge University Press, 1999), 375. 
- Protestation contre le refus par le Sate Département américain d'un visa pour Alberto Moravia.

- Envoi d'un message au Président Eisenhower demandant une mesure de clémence pour les Rosenberg.

- Publication par le Congrès d'un livre intitulé "McCarthy et les Communistes", exposant la nature du McCarthyisme.

- Protestation adressée à l'Université d'Alabama et au Gouverneur de l'État contre le renvoi de l'Université d'étudiant nègre.

Ces actions qui sont basés sur d'idéaux de justice et d'humaniste s'attaquent à des problèmes de politique interne américaine ou liée à la politique externe américaine. Même si ses actions en soi n'ont pas forcément été un succès sur le papier, il est clair qu'elles ont permis de diffuser certaines valeurs, une certaine approche des problématiques.

\section{Dynamique interne-externe et fabrication de fausses réalités}

Si la guerre froide fut un événement réel, elle produit aussi de fausses réalités. D'autres travaux ont cherché à savoir dans quelle mesure les intellectuels avaient participé à la création de ces fausses idées, ou du moins en avaient été les relais. ${ }^{33}$ Il semble y avoir eu un petit écosystème de débats idéologiques et littéraires qui ont soudainement acquis une audience internationale grâce à un soutien financier de la CIA.

La diffusion de ses idées a participé à la construction de la formation culturelle de ce qu'on appelle la "guerre froide». Cela a permis à l'URSS et aux USA de partager le sentiment d'être ennemis et de construire leur identité et intérêt pour chaque situation, reproduisant ainsi la guerre froide. ${ }^{34}$ Les États-Unis ont construit une partie de leur identité sur l'idéologie de liberté, en réaction à l'identité URSS perçue par les États-Unis. Dans le cas du congrès pour la liberté de la culture, la CIA a créé une structure permettant à des intellectuels européens compatibles avec l'idéologie américaine de promouvoir leurs idées avec des

\footnotetext{
${ }^{33}$ Saunders, The Cultural Cold War, xii.

${ }^{34}$ Wendt, Social Theory of International Politics, 187.
} 
moyens relativement importants. Mais cette structure, dont le but officiel était de défendre la liberté de la culture, sur des basses humanistes et de la liberté en général, s'en est aussi parfois prise aux positions politiques et aux actes des ÉtatsUnis.

Il y a donc une dynamique entre le système interne et externe. ${ }^{35}$ Il est souvent relativement facile de comprendre comment l'interne à une influence sur l'externe: ici le financement par la CIA permet de créer une structure anticommunisme basé sur l'idée de la liberté de la culture. Bien souvent les analyses politiques s'arrêtent là, sur l'action directe des États et leurs conséquences, ou sur les stratégies mises en place par d'autres États pour éviter telle ou telle situation. Mais l'externe affecte aussi l'interne: la politique extérieure des États-Unis et les pratiques reviennent pour influencer la politique interne des États-Unis.

\section{Bibliographie}

American Library Association, and American Book Publisher Council. "The Freedom to Read Statement," 1953.

“Appeal For Morton Sobell.” The Manchester Guardian, March 14, 1956.

"Bertrand Russel Accuses the FBI Of Atrocities, Doubts Rosenbergs' Guilt." The New York Times, March 26, 1965.

Bertrand Russell. "Letters to the Editor - The Sobell Case." The Manchester Guardian, March 26, 1956.

"Bertrand Russell Criticised." The Manchester Guardian, April 3, 1956.

"Campaign in U.S. to Pardon Negro." The Manchester Guardian, May 5, 1951.

Christel Lamboley. Le Maccarthysme Ou La Peur Rouge: La Croisade Américaine Contre Le Communisme. 50 Minutes, 2015.

David-Fox, Michael. Showcasing the Great Experiment: Cultural Diplomacy and Western Visitors to Soviet Union, 1921-1941. Oxford; New York: Oxford University Press, 2012.

"Dwight D. Eisenhower: 'Remarks at the Dartmouth College Commencement Exercises, Hanover, New Hampshire.," 1953. http://www.presidency.ucsb.edu/ws/?pid=9606.

François Chaubet, and Laurent martin. Histoire Des Relations Culturelles Dans Le Monde Contemporain. Armand Colin., 2011.

Hugh Wilford. The New York Intellectuals: From Vanguard to Institution. Manchester Univ Pr, 1995.

Lerner, K. Lee, and Brenda Wilmoth Lerner, eds. Encyclopedia of Espionage, Intelligence, and Security - Volume 2. Vol. 2. Detroit: Thomson/Gale, 2004.

Max Freedman. "The 'innocense' of Sobell - M. Sartre Gives His Views." The Manchester Guardian, June 16, 1956.

${ }^{35}$ Michael David-Fox, Showcasing the Great Experiment: Cultural Diplomacy and Western Visitors to Soviet Union, 1921-1941 (Oxford ; New York: Oxford University Press, 2012), 314. 
Michael Meeropol, and Robert Meeropol. "The Meeropol Brothers Exonerate Our Mother, Ethel Rosenberg." The New York Times, August 15, 2015.

Michael Warner. "Origins of the Congress for Cultural Freedom." Studies on Intelligence 38 (1995). https://www.cia.gov/library/center-for-the-study-of-intelligence/kentcsi/vol38no5/pdf/v38i5a10p.pdf.

"Negro's Execution in Mississippi." The Manchester Guardian, May 10, 1951.

"Russell Is Disputed." The New York Times, April 8, 1956.

Saunders, Frances Stonor. The Cultural Cold War: The CIA and the World of Arts and Letters. New Press, The, 2013.

Sidney Hook. Out of Step: An Unquiet Life in the 20th Century, 1987.

Wade . Mack. "Letters to the Editor - The Case Of Morton Sobell." The Manchester Guardian, April 3, 1956.

Wayne A. Wiegand. "The Freedom to Read - The History of ALA's Vital Statement on Intellectual Freedom.” American Libraries, March 15, 2016.

Wendt, Alexander. "Collective Identity Formation and the International State." American Political Science Review 88, no. 02 (June 1994): 384-96. doi:10.2307/2944711.

Social Theory of International Politics. Cambridge Studies in International Relations 67. Cambridge, UK ; New York: Cambridge University Press, 1999. 